\title{
Đánh giá hiệu quả quản trị hành chính công tại tỉnh Long An: Ứng dụng mô hình SEM
}

\section{Assessing the effectiveness of public administration in Long An province: An application of the SEM model}

\begin{tabular}{|c|c|}
\hline \multicolumn{2}{|c|}{$\begin{array}{l}{ }^{1} \text { Trường Đại học Mở Thành Phố Hồ Chí Minh, Việt Nam } \\
{ }^{*} \text { Tác giả liên hệ, Email: phuoc.nk@ ou.edu.vn }\end{array}$} \\
\hline THÔNG TIN & TÓM TẮT \\
\hline $\begin{array}{l}\text { DOI: } 10.46223 / \text { HCMCOUJS. } \\
\text { econ.vi.13.1.531.2018 }\end{array}$ & $\begin{array}{l}\text { Nghiên cứu ứng dụng mô hình SEM để đánh giá hiệu quả } \\
\text { quản trị hành chính công tại tỉnh Long An. Nghiên cứu sử dụng } \\
\text { thang đo likert } 9 \text { điểm. Mô hình nghiên cứu có } 4 \text { nhóm nhân tố } \\
\text { độc lập: Nguồn nhân lực, cơ sở vật chất, qui trình thủ tục hành } \\
\text { chính và đạo đức nghề nghiệp với } 27 \text { biến quan sát, dùng để kiểm } \\
\text { đinh mối quan hê với hiệu quả quản trì hành chính công (có } 5\end{array}$ \\
\hline Ngày nhận: 08/09/2017 & biến quan sát) tại tỉnh Long An. Kết quả phỏng vấn trực tiếp trong \\
\hline Ngày nhận lại: 02/10/2017 & 700 cán bộ công chức viên chức, doanh nghiệp, người dân đang \\
\hline Duyệt đăng: 20/12/2017 & $\begin{array}{l}\text { làm việc, kinh doanh và sinh sống tại các huyện/thị/thành phố } \\
\text { trong tỉnh, thu được } 672 \text { phiếu khảo sát đạt yêu cầu đưa vào phân } \\
\text { tích dữ liệu. Nghiên cứu sử dụng phương pháp phân tích nhân tố } \\
\text { khám phá (EFA), phân tích nhân tố khắng định (CFA) và mô } \\
\text { hình SEM. Kết quả nghiên cứu cho thấy, "nguồn nhân lực" và } \\
\text { "đạo đức nghề nghiệp" của cán bộ công chức viên chức là hai }\end{array}$ \\
\hline $\begin{array}{l}\text { Tì khóa: } \\
\text { cán bộ và công chức, Long } \\
\text { An, mô hình SEM, quản trị, } \\
\text { hành chính công }\end{array}$ & $\begin{array}{l}\text { nhân tố có ảnh hưởng mạnh, đóng vai trò quyết định đối với hiệu } \\
\text { quả quản trị hành chính công của tỉnh Long An. Dựa vào kết quả } \\
\text { nghiên cứu, tác giả đề xuất một số khuyến nghị chính sách nhằm } \\
\text { nâng cao hiệu quả quản trị hành chính công tại tỉnh Long An. }\end{array}$ \\
\hline
\end{tabular}

ABSTRACT
The study uses the SEM model to evaluate the effectiveness
of public administration in Long An province. The study
employs a 9-point Likert scale and four groups of independent
factors including human resources, facilities, administrative
procedures and professional ethics with 27 observation variables
to test the correlation between among these factors and the
effectiveness of public administration (5 variables) in Long An
province. Then, the study analyzes data collected from 672 valid
samples out of 700 interview sheets of officials, civil servants,


Keywords:

Long An province, management, offcials and public servants, public administration, SEM

employees and civilians working and living in the districts, towns and cities in the province using exploratory factor analysis (EFA), CFA and SEM models. The findings show that "human resources" and "professional ethics" of local officials and civil servants positively affect the effectiveness of public administration in Long An province. Based on the research results, the author makes some policy recommendations to improve the effectiveness of public administration in Long An province.

\section{Giới thiệu}

Chính phủ đã ban hành và thực hiện chương trình tổng thể cải cách hành chính nhà nước với nhiều kết quả đạt được khá tích cực. Mối quan hệ giữa Nhà nước với Nhân dân trong nền kinh tế thị trường đã được định hình, góp phần thúc đẩy tăng trưởng kinh tế, ổn định xã hội. Cải cách hành chính nhằm tổ chức, sắp xếp lại bộ máy nhà nước cho hoàn thiện, nâng cao hiệu quả hoạt động. Quá trình cải cách này đã thu được những kết quả tích cực như: thúc đẩy tính độc lập và chịu trách nhiệm trong quá trình ra quyết định, tổ chức thực hiện của chính quyền địa phương các cấp, giúp cải thiện môi trường kinh doanh; xây dựng và nâng cao chất lượng đội ngũ cán bộ công chức viên chức đáp ứng yêu cầu phát triển kinh tế thị trường định hướng xã hội chủ nghĩa và hội nhập quốc tế. Mặc dù cải cách hành chính đã được tiến hành trong thời gian không ngắn nhưng vẫn còn khá nhiều bất cập về tổ chức bộ máy, thủ tục và cơ chế, chính sách. Có nhiều nguyên nhân của những hạn chế nêu trên và một trong những nguyên nhân quan trọng là chưa đẩy mạnh cải cách hành chính theo hướng bảo đảm thực hiện ngày một tốt hơn quyền dân chủ của người dân và ngược lại, phát huy tinh thần dân chủ của người dân để nâng cao hiệu lực, hiệu quả của cải cách hành chính (Chính phủ, 2011).

Cùng với sự phát triển của cả nước, chính quyền tỉnh Long An thời gian qua đã có nhiều nỗ lực, thực hiện nhiều giải pháp để cải cách hành chính nhằm phục vụ người dân ngày một tốt hơn. Bên cạnh những kết quả đạt được rất khả quan, Long An cũng là địa phương còn nhiều tồn tại, hạn chế trong quá trình giải quyết công việc cho tổ chức, công dân ở một số cơ quan, đơn vị chưa tuân thủ đầy đủ quy trình, còn đặt thêm giấy tờ ngoài quy định thủ tục hành chính; còn hồ sơ trễ hẹn... (Ủy ban nhân dân tỉnh Long An, 2016). Với mục tiêu góp phần xây dựng chính quyền địa phương năng động, hiệu quả tạo điều kiện thúc đẩy hơn nữa cho phát triển kinh tế, xã hội của tỉnh... Nghiên cứu "Đánh giá hiệu quả quản trị hành chính công tại tỉnh Long $A n$ " được thực hiện nhằm phân tích các nhân tố ảnh hưởng đến hiệu quả quản trị hành chính công tại tỉnh Long An, để từ đó giúp chính quyền có những giải pháp phù hợp, thúc đẩy nhanh và hiệu quả quá trình cải cách hành chính công tại địa phương.

Kết cấu của nghiên cứu này gồm: Phần 2 trình bày về cơ sở lý luận; Phần 3 trình bày về mô hình và phương pháp nghiên cứu; Phần 4 trình bày kết quả nghiên cứu; Phần 5 gồm kết luận và khuyến nghị. 


\section{Cơ sở lý luận}

Nguyen (2005) cho rằng, các hoạt động thuộc khu vực công là các hoạt động thuộc các lĩnh vực: (i) Xây dựng, hoàn thiện, thực hiện và duy trì hệ thống luật pháp của quốc gia một cách có hiệu quả. Đây là hoạt động rất quan trọng thuộc khu vực công và cũng chỉ tồn tại ở khu vực công. (ii) Các hoạt động dưới dạng các chính sách của Chính phủ như các chính sách về thuế, phạt tiền, chính sách về trợ cấp, hệ thống các quy định tiêu chuẩn về môi trường, về an toàn thực phẩm, về quy định đô thị và về vệ sinh lao động... (iii) Các hoạt động về cung ứng dịch vụ, sản xuất hàng hóa công cộng.

Le (2006) định nghĩa, hành chính công là hoạt động của Nhà nước, của các cơ quan Nhà nước mang tính quyền lực Nhà nước, sử dụng quyền lực Nhà nước để quản lý công việc công của Nhà nước nhằm phục vụ lợi ích chung hay lợi ích riêng hợp pháp của công dân. Do vậy, hành chính công bao hàm toàn bộ các cơ quan thuộc chính quyền của bộ máy hành pháp từ trung ương tới các cấp chính quyền địa phương, toàn bộ các thể chế và hoạt động của bộ máy ấy và tất cả những người làm việc trong bộ máy đó.

Theo Pham (2012), hành chính công tổng quát bao gồm các yếu tố: Hệ thống thể chế, pháp luật, thủ tục hành chính; chức năng, nhiệm vụ, cơ cấu tổ chức và cơ chế vận hành của bộ máy hành chính nhà nước các cấp; đội ngũ cán bộ, công chức; nguồn lực tài chính, công sản và các điều kiện vật chất, kỹ thuật đảm bảo thực thi công vụ hiệu quả. Trong nghiên cứu này, các yếu tố hành chính công được chọn để đánh giá là: Thủ tục hành chính, đội ngũ cán bộ, công chức; các điều kiện vật chất.

Hiệu quả quản trị liên quan đến việc hoàn thành mục tiêu công việc của tổ chức với việc sử dụng các nguồn lực (nhân lực, tài lực, trang thiết bị) một cách có hiệu quả (Peter, 2016). Quản trị hành chính công là những công việc hành chính, quản lý vận hành các hoạt động hành chính của các cơ quan chính phủ và các tổ chức thuộc khu vực công (Raadschelders, 2011).

Từ năm 2009 đến nay, CECODES và UNDP tại Việt Nam phối hợp cùng Mặt Trận Tổ Quốc Việt Nam đưa ra chỉ số hiệu quả quản trị và hành chính công cấp tỉnh (PAPI) nhằm đánh đo lường và phản ánh năng lực và hiệu quả quản trị ở cấp trung ương và cấp tỉnh (CECODES, 2012). Chỉ số PAPI bao gồm 6 chỉ số lĩnh vực nội dung, 22 chỉ số nội dung thành phần và 92 chỉ tiêu thành phần về hiệu quả quản trị và hành chính công. Tuy nhiên, chỉ số này tập trung quá nhiều chỉ tiêu ở hộ nghèo, tri thức công dân, thanh tra nhân dân, bầu cử, ... số chỉ tiêu tập trung đánh giá về hành chính công chưa nhiều, cụ thể là chỉ có 11/92 chỉ số đánh giá về hành chính công ${ }^{1}$ trong đó tập trung vào dịch vụ công là chủ yếu.

Theo Hughes (2012), quản lý hành chính công theo phương thức truyền thống (xếp theo hệ thống luật pháp và các qui định địa giới hành chính) làm cho các hoạt động thực thi các quy định một cách kém linh hoạt. Mô hình quản lý hành chính công truyền thống lấy tổ chức thứ bậc chặt chẽ, trình tự, thủ tục và việc thực hiện các thủ tục đó theo quy trình mà ít quan tâm đến kết quả hoạt động của nhà nước hoặc các cơ quan, tổ chức khu vực công.

\footnotetext{
${ }^{1}$ Xem thêm chỉ số PAPI tại http://papi.org.vn/chi-so
} 
Theo Ocampo (1998), nhiều quốc gia ứng dựng mô hình mới về quản lý công đã cho thấy kết quả rất tốt. Kết quả đã được đúc kết thành tám đặc trưng về quản lý công mới là: (1) tăng cường chức năng chỉ đạo ở trung ương; (2) phân cấp thẩm quyền, cung cấp linh hoạt; (3) bảo đảm thực hiện, kiểm soát, trách nhiệm; (4) cải thiện quản lý nguồn nhân lực; (5) tối ưu hóa công nghệ thông tin; (6) phát triển cạnh tranh và sự lựa chọn; (7) nâng cao chất lượng quy định; và (8) cung cấp dịch vụ đáp ứng.

Theo Hood (1991), cải cách hành chính công dựa trên nền tảng thị trường được xem như một điểm đặc trưng của quản trị công mới. Điểm khác biệt mấu chốt trong quản lý hành chính công mới là nhà quản lý công chịu trách nhiệm cá nhân với các kết quả đạt được.

Robert, Janet, và Tara (2014) đưa ra khung phân tích hiệu quả hành chính công gồm 3 cấp độ: (i: cấp độ 1) bao gồm các hiện vật, cơ sở vật chất, các kiến trúc có thể nhìn thấy được như là không gian làm việc, phong cách trang phục, sản phẩm, công nghệ, lưu trữ của tổ chức; (ii: cấp độ 2) bao gồm các giá trị và hành vi như ngôn ngữ, hành vi, thói quen, và các quá trình của cơ quan (có thể quan sát và nhận biết được; (iii: cấp độ 3) là giá trị đạo đức, những niềm tin, mục đích và triết lý của một tổ chức, những nhận thức, suy nghĩ và cảm xúc phục vụ của nhân viên, quản lý trong tổ chức đó.

Quản trị công mới, theo Hood (1991), biểu thị một cách rộng rãi các chính sách của Chính phủ nhằm mục đích hiện đại hóa và tăng cường hiệu quả khu vực công. Quản trị công mới thường được sử dụng khi nói đến "Mô hình hành chính công theo các tiêu chí hiện đại, chủ động, năng động, nhạy bén, thích nghi cao nhằm đáp ứng tối đa các yêu cầu quản lý và hiệu quả cung cấp dịch vụ công trong các điều kiện kinh tế thị trường phát triển mạnh mẽ và bối cảnh quan hệ quốc tế ngày càng phụ thuộc chặt chẽ lẫn nhau". Dựa trên những nền tảng lý thuyết về quản lý công mới, một số nghiên cứu được thực hiện như:

Viện nghiên cứu đổi mới kinh tế Trung ương (2007) nghiên cứu mô hình quản lý theo kết quả tại Thành phố Hồ Chí Minh. Sau khi triển khai thí điểm tại 3 Sở và 2 quận/huyện của Thành phố Hồ Chí Minh, kết quả nghiên cứu cho thấy, việc thực hiện hệ thống quản lý theo kết quả được coi là khâu đột phá mới trong công tác hành chính nhà nước và đã thu được những kết quả rất khả quan, sự hài lòng của người dân và doanh nghiệp được nâng cao. Viện nghiên cứu đổi mới kinh tế Trung ương (2007) nhận định, yếu tố con người (CBCCVC) vẫn là yếu tố quyết định hiệu quả quản trị hành chính công.

Pham (2012) nghiên cứu mô hình xây dựng cơ cấu cán bộ, công chức các cơ quan chuyên môn thuộc Ủy ban nhân dân cấp huyện, cấp tỉnh của Bắc Ninh nhằm hoàn thiện hơn nữa công tác tổ chức và quản lý cán bộ, công chức, viên chức ở địa phương. Kết quả nghiên cứu cho thấy, xây dựng quy trình làm việc, cải cách thủ tục hành chính, xác lập được quy trình làm việc khoa học, hiệu quả là yếu tố quyết định. Qua đó, các thủ tục hành chính cũng được xác định rõ ràng, công khai, minh bạch, thuận tiện cho việc áp dụng cơ chế "một cửa" tại các cơ quan, đơn vị.

Nozharov (2014) xây dựng một mô hình hiệu quả quản lý công ở Bungari trong quản lý các quỹ của $\mathrm{EU}$ trong bối cảnh khủng hoảng tài chính toàn cầu nhằm mục đích tăng khả năng thu hút vốn ở các quỷ đầu tư của UU vào Bulgaria. Phân tích cũng giúp cho Chính phủ Bulgaria xác định rõ một số điểm yếu trong cơ cấu tổ chức quản lý hành chính gây ảnh hưởng đến quá 
trình thu hút và quản lý quỹ đầu tư. Kết quả nghiên cứu cho thấy, sự phối hợp quản lý của các cơ quan quản lý, sự trùng lặp trong các qui trình thủ tục và vấn đề tham nhũng là những yếu tố có ảnh hưởng đến hiệu quả quản lý công ở Bungari.

Nedelko (2015) nghiên cứu các giá trị, thái độ và đạo đức của cá nhân và nhà quản lý ảnh hưởng với môi trường tự nhiên - xã hội trong quản lý hành chính công của chính quyền Slovenia. Mẫu nghiên cứu gồm bảng khảo sát 212 nhà quản lý của chính quyền Slovenia với thang đo likert 9 điểm (các thang đo đưa vào khảo sát áp dụng theo Schwartz, 1992). Tác giả chọn mẫu dựa trên lựa chọn các đơn vị hành chính (MOPA, 2014) và ưu tiên chọn những đơn vị hành chính gần với mục tiêu nghiên cứu và có qui mô lớn. Mô hình nghiên cứu gồm có: nhóm yếu tố đạo đức của nhân viên và quản lý, và thái độ phục vụ của nhân viên và quản lý. Kết quả nghiên cứu cho thấy, các nhóm biến quan sát đều có ý nghĩa thống kê, nghĩa là có tác động đến quản lý hành chính công của chính quyền Slovenia. Trong đó, yếu tố đạo đức của nhân viên và quản lý có ảnh hưởng mạnh nhất.

Nozharov (2017) nghiên cứu về hiệu quả quản trị công trong bảo vệ di sản văn hóa được UNESCO công nhận ở Bulgaria. Mục tiêu chính của nghiên cứu này là đề xuất các biện pháp cho phát triển quản lý và kiểm soát hiệu quả của việc bảo vệ di sản văn hóa do chính quyền hành chính thực hiện (Bộ Văn hóa Bungari, 2017). Tác giả dựa vào khung phân tích bảy bước của Kaplan (2005). Nghiên cứu đã rút ra kết luận là số nhân viên chịu trách nhiệm, trình độ nhân viên, phong cách quản lý, hệ thống và cơ cấu của cơ quan quản lý, hiệu quả của nguồn nhân lực có ảnh hưởng mạnh đến hiệu quả quản lý công trong bảo vệ di sản văn hóa.

\section{Mô hình và phương pháp nghiên cứu}

\subsection{Mô hình nghiên cứu}

Dựa vào cơ sở lý thuyết và các nghiên cứu trước đã được trình bày như trên, qua tìm hiểu tình hình thực tế và thảo luận với các chuyên gia tại địa bàn nghiên cứu. Mô hình nghiên cứu đánh giá hiệu quả quản trị hành chính công tại tỉnh Long An được đề nghị như sau:

$$
\mathrm{Y}=\beta_{0}+\beta_{1} * \mathrm{CSVC}+\beta_{2} * \mathrm{NNL}+\beta_{3} * \mathrm{DDNN}+\beta_{4} * \mathrm{QQTT}+\varepsilon
$$

Trong đó:

- Biến phụ thuộc (Y): Hiệu quả quản trị hành chính công (5 biến quan sát);

- Các biến độc lập:

+ CSVC - Cơ sở vật chất kỹ thuật (4 biến);

+ NNL - Nguồn nhân lực hay đội ngũ cán bộ, công chức, viên chức (7 biến);

+ DDNN - Đạo đức nghề nghiệp ( 9 biến);

+ QTTT - Quy trình, thủ tục hành chính (7 biến).

- $\beta 0$ : Hệ số gốc;

- $\beta_{1}, \beta_{2}, \beta_{3}, \beta_{4}$ : Các hệ số hồi quy;

- E: Sai số. 


\section{Bảng 1}

Cơ sở chọn biến và kỳ vọng dấu

\begin{tabular}{|c|c|c|c|}
\hline Biến quan sát & $\begin{array}{l}\text { Nhóm } \\
\text { nhân tố }\end{array}$ & $\begin{array}{l}\text { Nghiên cứu } \\
\text { trước }\end{array}$ & $\begin{array}{l}\text { Kỳ vọng } \\
\text { dấu }\end{array}$ \\
\hline Trụ sở làm việc (CSVC1) & \multirow{4}{*}{$\begin{array}{l}\text { Cơ sở vật } \\
\text { chất } \\
\text { (CSVC) }\end{array}$} & \multirow{4}{*}{$\begin{array}{c}\text { Hood (1991), } \\
\text { Ocampo } \\
\text { (1998), } \\
\text { Hughes } \\
\text { (2012), } \\
\text { Robert và } \\
\text { cộng sự } \\
\text { (2014) }\end{array}$} & \multirow{4}{*}{$\begin{array}{l}\text { Dương } \\
(+)\end{array}$} \\
\hline Vị trí trụ sở làm việc (CSVC2) & & & \\
\hline $\begin{array}{l}\text { Phương tiện, thiết bị văn phòng đảm bảo phục vụ tốt } \\
\text { (CSVC3) }\end{array}$ & & & \\
\hline $\begin{array}{l}\text { Có trang Web (trang mạng) tra cứu tiến độ giải quyết } \\
\text { hồ sơ (CSVC4) }\end{array}$ & & & \\
\hline $\begin{array}{l}\text { Kỹ năng và kinh nghiệm giải quyết công việc của cán } \\
\text { bộ (NNL1) }\end{array}$ & \multirow{7}{*}{$\begin{array}{c}\text { Nguồn } \\
\text { nhân lực/ } \\
\text { CBCCVC } \\
\text { (NNL) }\end{array}$} & \multirow{7}{*}{$\begin{array}{c}\text { Ocampo } \\
(1998), \\
\text { Nozharov } \\
(2017)\end{array}$} & \multirow{7}{*}{$\begin{array}{l}\text { Dương } \\
\qquad(+)\end{array}$} \\
\hline Thái độ làm việc của cán bộ (NNL2) & & & \\
\hline $\begin{array}{l}\text { CB có khả năng lắng nghe và tiếp thu ý kiến phản ánh } \\
\text { (NNL3) }\end{array}$ & & & \\
\hline $\begin{array}{l}\text { CB giải quyết công việc linh hoạt, phù hợp với quy } \\
\text { định của pháp luật (NNL4) }\end{array}$ & & & \\
\hline $\begin{array}{l}\text { Thái độ của CB tạo cảm giác thân thiện và tin tưởng } \\
\text { vào cơ quan nhà nước (NNL5) }\end{array}$ & & & \\
\hline $\begin{array}{l}\text { CB sẵn sàng giải đáp những vướng mắc trong công } \\
\text { việc (NNL6) }\end{array}$ & & & \\
\hline $\begin{array}{l}\text { Khả năng ứng dụng công nghệ thông tin của CBCCVC } \\
\text { (NNL7) }\end{array}$ & & & \\
\hline Công khai thủ tục hành chính (QTTT1) & \multirow{7}{*}{$\begin{array}{l}\text { Qui trình } \\
\text { thủ tục } \\
\text { (QTTT) }\end{array}$} & \multirow{7}{*}{$\begin{array}{c}\text { Hughes } \\
(2012), \\
\text { Nozharov } \\
\text { (2014) }\end{array}$} & \multirow{7}{*}{$\begin{array}{c}\text { Dương } \\
(+)\end{array}$} \\
\hline Trình tự giải quyết hồ sơ (QTTT2) & & & \\
\hline Thời hạn trả hồ sơ trả theo qui định (QTTT3) & & & \\
\hline Các yêu cầu được giải quyết theo qui định (QTTT4) & & & \\
\hline Tình trạng đơn giản hóa thủ tục hành chính (QTTT5) & & & \\
\hline Hồ sơ được giải quyết nhanh chóng (QTTT6) & & & \\
\hline Khả năng phối hợp giải quyết hồ sơ (QTTT7) & & & \\
\hline $\begin{array}{l}\text { Ngăn chặn tình trạng "hạch sách, nhũng nhiễu, lót tay, } \\
\text { vòi vĩnh, tham ô, hối lộ" ở cơ quan/địa phương } \\
\text { (DDNN1) }\end{array}$ & \multirow{2}{*}{$\begin{array}{l}\text { Đạo đức } \\
\text { Nghề } \\
\text { nghiệp của }\end{array}$} & \multirow{2}{*}{$\begin{array}{l}\text { Nozharov } \\
\text { (2017), } \\
\text { Nedelko } \\
(2015)\end{array}$} & \multirow{2}{*}{$\begin{array}{c}\text { Dương } \\
(+)\end{array}$} \\
\hline Tình trạng lợi dụng chức quyền (DDNN2) & & & \\
\hline
\end{tabular}




\begin{tabular}{|c|c|c|c|}
\hline Biến quan sát & $\begin{array}{l}\text { Nhóm } \\
\text { nhân tố }\end{array}$ & $\begin{array}{l}\text { Nghiên cứu } \\
\text { trước }\end{array}$ & $\begin{array}{l}\text { Kỳ vọng } \\
\text { dấu }\end{array}$ \\
\hline $\begin{array}{l}\text { Ngăn chặn tình trạng “đưa và nhận hối lộ” ở cơ } \\
\text { quan/địa phương (DDNN3) }\end{array}$ & \multirow[t]{7}{*}{$\begin{array}{l}\text { CBCCVC } \\
(\mathrm{DDNN})\end{array}$} & & \\
\hline $\begin{array}{l}\text { Ngăn chặn tình trạng “thiên vị” khi giải quyết công } \\
\text { việc/hồ sơ (DDNN4) }\end{array}$ & & & \\
\hline $\begin{array}{l}\text { Tình trạng xử lý "hạch sách, nhũng nhiễu, lót tay, vòi } \\
\text { vĩnh, tham ô, hối lộ" ở cơ quan/địa phương (DDNN5) }\end{array}$ & & & \\
\hline $\begin{array}{l}\text { Ngăn ngừa tình trạng việc bao che cho CB khi sai } \\
\text { phạm (DDNN6) }\end{array}$ & & & \\
\hline $\begin{array}{l}\text { Dễ dàng gặp gỡ, trao đổi với lãnh đạo cấp cao } \\
\text { (DDNN7) }\end{array}$ & & & \\
\hline Tình trạng đùn đẩy trách nhiệm (DDNN8) & & & \\
\hline Tác phong, ngôn phong của CB công chức (DDNN9) & & & \\
\hline Đánh giá chung về CSVC (HQQT1) & \multirow{5}{*}{$\begin{array}{l}\text { Hiệu quả } \\
\text { quản trị } \\
\text { hành chính } \\
\text { công } \\
\text { (HQQT) }\end{array}$} & \multirow{5}{*}{$\begin{array}{l}\text { Viện nghiên } \\
\text { cứu đổi mới } \\
\text { kinh tế Trung } \\
\text { ương (2007) }\end{array}$} & \multirow{5}{*}{$\begin{array}{l}\text { Nhóm biến } \\
\text { phụ thuộc }\end{array}$} \\
\hline Đánh giá chung CBCCVC - NNL (HQQT2) & & & \\
\hline Đánh giá chung về ĐĐNN (HQQT3) & & & \\
\hline Đánh giá chung về qui trình thủ tục HCC (HQQT4) & & & \\
\hline $\begin{array}{l}\text { Đánh giá chung về công tác quản lý hành chính } \\
\text { (HQQT5) }\end{array}$ & & & \\
\hline
\end{tabular}

Nguồn: Kết quả phân tích dữ liệu của nhóm nghiên cứu

\subsection{Phương pháp nghiên cúu}

Nghiên cứu được thực hiện thông qua hai giai đoạn: Nghiên cứu định tính và nghiên cứu định lượng. Nghiên cứu định tính được thực hiện bằng cách thảo luận nhóm với một số chuyên gia làm việc ở các cơ quan ban ngành cấp tỉnh và các địa phương trong tỉnh Long An để hoàn thiện bảng câu hỏi khảo sát. Nghiên cứu chính thức được thực hiện bằng phương pháp định lượng: phỏng vấn trực tiếp các cán bộ công chức viên chức, doanh nghiệp và người dân đang làm việc, cư trú trên địa bàn nghiên cứu đề tạo lập dữ liệu sơ cấp. Đề tài sử dụng dữ liệu sơ cấp qua việc phỏng vấn trực tiếp 700 cán bộ, công chức bằng bảng câu hỏi soạn sẵn. Bảng câu hỏi khảo sát được thiết kế theo thang đo Likert 9 điểm, cụ thể là: Mức 1: Rất kém; Mức 2: Kém; Mức 3: Yếu kém; Mức 4: Yếu; Mức 5: Trung bình; Mức 6: Trung bình khá; Mức 7: Khá (khá tốt); Mức 8: Giỏi (rất tốt); Mức 9: Hoàn toàn rất tốt. Nghiên cứu sử dụng thang điểm Likert 9 điểm nhằm gia tăng mức đánh giá để người tham gia khảo sát đánh giá một cách cẩn trọng và chính xác hơn (Ralston et al., 2011). Theo Schwartz (1992), thang đo Likert 9 điểm thường được dùng trong các khảo sát về quản trị hành chính công vì khu vực công có xu hướng thay đổi nhanh theo sự thay đổi của chính trị - xã hội.

Nghiên cứu sử dụng phương pháp phân tích nhân tố khẳng định $(\mathrm{CFA})$ và mô hình cấu trúc tuyến tính (SEM). Mô hình SEM có nhiều ưu điểm hơn những mô hình phân tích thống kê truyền thống như phân tích khám phá EFA, tương quan và hồi quy đa biến (Bagozzi \& Youjae, 
1989). CFA cho phép chúng ta kiểm định cấu trúc lý thuyết của các thang đo như mối quan hệ của khái niệm nghiên cứu với các khái niệm khác mà không bị chệch (Steenkamp \& Van Trijp, 1991). SEM cho phép chúng ta kết hợp các nhân tố (khái niệm tiềm ẩn) với các biến quan sát (khái niệm đo lường). Đồng Thời, SEM được sử dụng nhiều trong đánh giá thang đo dịch vụ áp dụng thang đo Likert vì nó cho phép đánh giá được độ phù hợp của mô hình, kiểm định được giá trị hội tụ và giá trị phân biệt mà không cần qua nhiều bước kiểm định và hồi quy (Hulland, Chow, \& Lam, 1996).

Theo MOPA (2014) để đảm bảo tính đại diện và sự tin cậy của dữ liệu thu thập, chọn mẫu theo hướng ưu tiên chọn những đơn vị hành chính gần với mục tiêu nghiên cứu và có qui mô lớn. Theo Nedelko (2015) để đánh giá hành chính công của các quốc gia, đối tượng thu thập ý kiến là nhà quản lý của chính quyền vì hơn ai hết, nhóm đối tượng này hiểu rõ nhất về hiệu quả quản lý của mình. Tuy nhiên, nếu theo quan điểm của Nedelko (2015) thì thiếu tính khách quan trong kết quả đánh giá. Do đó, trong nghiên cứu này, thực hiện thu thập ý kiến của các nhà quản lý của chính quyền cụ thể cán bộ công chức viên chức (đại diện cho đơn vị cung ứng), doanh nghiệp và người dân (đại diện cho người sử dụng). Mẫu khảo sát cũng lựa chọn nhưng đơn vị hành chính chính yếu của tỉnh Long An có qui mô lớn, nhiều người liên hệ như: Văn phòng UBND tỉnh, Sở Tài nguyên Môi trường, Sở Kế hoạch và Đầu tư, UBND thành phố Tân An, UBND thị xã Kiến Tường, ...

Mẫu được chọn theo phương pháp thuận tiện và đảm bảo nguyên tắc đánh giá chéo (đánh giá nơi có liên hệ công tác thường xuyên và không phải nơi công tác của mình). Quá trình khảo sát dữ liệu phục vụ cho nghiên cứu có 700 người tham gia trả lời phiếu khảo sát. Qua kiểm tra, loại bỏ các phiếu khảo sát trả lời thiếu thông tin, kết quả có 697 phiếu đầy đủ thông tin khảo sát. Trong số đó, chỉ có 3 phiếu đánh giá cho Công An tỉnh, 2 phiếu đánh giá Sở Giao thông vận tải, 4 phiếu đánh giá cơ quan thuế, 10 phiếu đánh giá Sở Công Thương và 6 phiếu đánh giá Sở Tư pháp. Số lượng người đánh giá các cơ quan này quá nhỏ (dưới 30) các phiếu này bị loại ra, không đưa vào mô hình phân tích dữ liệu nhằm đảm bảo tính tương đồng giữa các nghiên cứu (các cơ quan được đánh giá). Kết quả còn lại 672 phiếu khảo sát được lựa chọn để tiến hành phân tích dữ liệu.

\section{Phân tích kết quả nghiên cứu}

\subsection{Phân tích thống $k \hat{e}$ mô tả}

\section{Bảng 2}

Đơn vị hành chính được các đối tượng chọn đánh giá

\begin{tabular}{|c|l|c|c|}
\hline STT & \multicolumn{1}{|c|}{ Đơn vị } & Số lượng & Tỷ lệ (\%) \\
\hline 1 & VP UBND tỉnh & 47 & 7,0 \\
\hline 2 & Sở KH\&ĐT & 49 & 7,3 \\
\hline 3 & Sở Tài Chính & 39 & 5,8 \\
\hline 4 & Sở TNMT & 68 & 10,1 \\
\hline 5 & Sở Xây dựng & 37 & 5,5 \\
\hline
\end{tabular}




\begin{tabular}{|c|l|c|c|}
\hline STT & \multicolumn{1}{|c|}{ Đơn vị } & Số lượng & Tỷ lệ (\%) \\
\hline 6 & VP UBND \& PB Tp. Tân An & 103 & 15,3 \\
\hline 7 & VP UBND \& PB TX. Kiến Tường & 90 & 13,4 \\
\hline 8 & VP UBND \& PB H. Châu Thành & 69 & 10,3 \\
\hline 9 & VP UBND \& PB H. Đức Hòa & 104 & 15,5 \\
\hline 10 & VP UBND \& PB H. Cần Giuộc & 66 & 9,8 \\
\hline \multicolumn{2}{|l|}{ Tổng cộng } & $\mathbf{6 7 2}$ & $\mathbf{1 0 0}$ \\
\hline
\end{tabular}

Nguồn: Kết quả tính toán của tác giả

Đức Hòa là đơn vị được chọn để đánh giá nhiều nhất với 104 người, chiếm tỷ lệ 15,5\%, Tân An có 103 người chọn để đánh giá chiếm 15,3\%, tiếp theo là Kiến Tường. Về phía ban ngành cấp tỉnh, văn phòng UBND tỉnh, Sở Kế hoạch và Đầu tư, Sở Tài nguyên Môi trường là những đơn vị được nhiều người chọn để đánh giá. Đây cũng là những cơ quan được nhiều người liên hệ công tác, giao dịch hành chính. Do đó, số lượng mẫu đánh giá mang tính đại diện.

\section{Bảng 3}

Mẫu nghiên cứu phân theo nhóm đối tượng khảo sát

\begin{tabular}{|c|l|c|c|}
\hline STT & \multicolumn{1}{|c|}{ Nhóm đối tưọng khảo sát } & Số lượng & Tỷ lệ (\%) \\
\hline 1 & Không có việc làm/nghỉ hưu & 2 & 0,3 \\
\hline 2 & Chủ DN, tự SXKD & 311 & 46,3 \\
\hline 3 & Công nhân, LĐPT & 14 & 2,1 \\
\hline 4 & CBCCVC cấp huyện/thị/tp & 186 & 27,7 \\
\hline 5 & CBCCVC cấp xã/phường & 84 & 12,5 \\
\hline 6 & CBCCVC cấp tỉnh/sở ngành tỉnh & 57 & 8,5 \\
\hline 7 & Khác & 18 & 2,7 \\
\hline & Tổng số & 672 & 100,0 \\
\hline
\end{tabular}

Nguồn: Kết quả tính toán của tác giả

Số đối tượng tham gia trong mẫu khảo sát phân bổ khá tương đồng, mẫu gồm có đầy đủ các thành phần: doanh nghiệp, cán bộ công chức viên chức (CBCCVC) các cấp, lao động phổ thông. Các thành phần tham gia trong mẫu khảo sát đảm bảo tính đại diện cho các thành phần kinh tế. Điều này giúp cho kết quả đánh giá đảm bảo tính đại diện. 


\section{2. Đánh giá độ tin cậy của thang đo}

\section{Bảng 4}

Tổng hợp kết quả phân tích độ tin cậy của thang đo

\begin{tabular}{|l|c|c|c|c|}
\hline \multicolumn{1}{|c|}{ Nhân tố } & $\begin{array}{c}\text { Số biến } \\
\text { quan sát } \\
\text { ban đầu }\end{array}$ & $\begin{array}{c}\text { Số biến } \\
\text { quan sát sau } \\
\text { phân tích }\end{array}$ & $\begin{array}{c}\text { Hệ số } \\
\text { Cronbach's } \\
\text { Alpha }\end{array}$ & $\begin{array}{c}\text { Hệ số tương } \\
\text { quan biến tống } \\
\text { thấp nhất }\end{array}$ \\
\hline Cơ sở vật chất (CSVC) & 4 & 4 & 0,800 & 0,543 \\
\hline Nguồn nhân lực (NNL) & 7 & 7 & 0,851 & 0,396 \\
\hline Quy trình thủ tục (QTTT) & 7 & 6 & 0,908 & 0,591 \\
\hline Đạo đức nghề nghiệp (DDNN) & 9 & 9 & 0,927 & 0,508 \\
\hline Hiệu quả quản trị HCC (HQQT) & 5 & 5 & 0,965 & 0,871 \\
\hline
\end{tabular}

Nguồn: Kết quả tính toán của tác giả

Theo Hoang Trong và Chu (2008), hệ số Cronbach's Alpha của thang đo lớn hơn 0,8 nghĩa là thang đo tốt. Đồng thời, hệ số các hệ số tương quan đều lớn hơn 0,3 đạt yêu cầu. Trong quá trình đánh giá độ tin cậy của các thang đo, biến QTTT4 có hệ số tương quan biến tổng nhỏ hơn 0,3 nên đã loại biến này. Tất cả các biến quan sát trong các nhóm nhân tố còn lại đều đạt yêu cầu về hệ số tương quan biến tổng. Đồng thời, hệ số Cronbach's Alpha của các nhóm nhân tố đều $\geq 0,8$, nên có thể nói là thang đo sử dụng trong nghiên cứu là thang đo tốt, đảm bảo độ tin cậy cao.

Bước tiếp theo là đánh giá độ tin cậy của thang đo bằng phân tích nhân tố khám phá (EFA). Theo Hoang Trong và Chu (2008), điều kiện cần đạt được khi phân tích EFA để có được thang đo lường tốt là: hệ số $\mathrm{KMO} \geq 0,6$; Tổng $\%$ phương sai $\geq 50 \%$; Eigenvalues $\geq 1$, số biến quan sát trong cùng 1 nhân tố $\geq 2$; hệ số tải nhân tố (Factor loading) $\geq 0,5$. Kết quả sau hai lần phân tích EFA, các biến QTTT3, DDNN8, DDNN9, NNL6, NNL7 bị loại ra khỏi mô hình vì không đạt hệ số tải nhân tố như yêu cầu. Từ 32 biến quan sát của 5 nhóm nhân tố ban đầu, sau phân tích độ tin cậy có 6 biến quan sát bị loại, còn lại 26 biến tập trung vào 4 nhóm độc lập và 1 nhóm nhóm phụ thuộc (Bảng 5).

\section{Bảng 5}

Thống kê mô tả chung và ma trận tương quan các nhân tố sau phân tích EFA

\begin{tabular}{|l|l|l|l|l|l|l|l|l|}
\hline Nhân tố & Biến & $\begin{array}{c}\text { Trung } \\
\text { bình }\end{array}$ & $\begin{array}{c}\text { Độ̣ } \\
\text { lệch } \\
\text { chuẩn }\end{array}$ & CSVCF & QTTTF & NNLF & DDNNF & HQQTF \\
\hline CSVCF & $\begin{array}{l}\text { CSVC1 } \\
\text { CSVC2 } \\
\text { CSVC3 } \\
\text { CSVC4 }\end{array}$ & 6,944 & 1,635 & 1 & & & & \\
\hline \multirow{2}{*}{ QTTTF } & $\begin{array}{l}\text { QTTT1 } \\
\text { QTTT2 }\end{array}$ & 6,263 & 1,843 & $0,601^{* *}$ & 1 & & & \\
\hline
\end{tabular}




\begin{tabular}{|c|c|c|c|c|c|c|c|c|}
\hline Nhân tố & Biến & $\begin{array}{c}\text { Trung } \\
\text { bình }\end{array}$ & $\begin{array}{c}\text { Độ } \\
\text { lệch } \\
\text { chuẩn }\end{array}$ & CSVCF & QTTTF & NNLF & DDNNF & HQQTF \\
\hline & $\begin{array}{l}\text { QTTT5 } \\
\text { QTTT6 } \\
\text { QTTT7 }\end{array}$ & & & & & & & \\
\hline NNLF & $\begin{array}{l}\text { NNL1 } \\
\text { NNL2 } \\
\text { NNL3 } \\
\text { NNL4 } \\
\text { NNL5 } \\
\end{array}$ & 6,553 & 1,797 & $0,080^{*}$ & $0,251^{* *}$ & 1 & & \\
\hline DDNNF & $\begin{array}{l}\text { DDNN1 } \\
\text { DDNN2 } \\
\text { DDNN3 } \\
\text { DDNN4 } \\
\text { DDNN5 } \\
\text { DDNN6 } \\
\text { DDNN7 }\end{array}$ & 5,462 & 1,646 & 0,055 & $0,218^{* *}$ & $0,778 *$ & 1 & \\
\hline HQQTF & $\begin{array}{l}\text { HQQT1 } \\
\text { HQQT2 } \\
\text { HQQT3 } \\
\text { HQQT4 } \\
\text { HQQT5 }\end{array}$ & 5,978 & 1,301 & 0,076 & $0,227 *$ & $0,862^{* *}$ & $0,795^{*}$ & 1 \\
\hline
\end{tabular}

Nguồn: Kết quả tính toán của tác giả

Thống kê mô tả chung và ma trận tương quan các nhân tố sau phân tích EFA (Bảng 5) cho thấy, nhóm "đạo đức nghề nghiệp" có giá trị trung bình thấp nhất, nhóm "cơ sở vật chất" có giá trị trung bình cao nhất. Nhóm "qui trình thủ tục" và "nguồn nhân lực" là hai nhóm đánh giá không đồng đều giữa các đối tượng tham gia khảo sát thể hiện qua độ lệch chuẩn cao. Kết quả phân tích tương quan cho thấy, các nhóm nhân tố có tương quan đồng biến với nhau và có ý nghĩa với hiệu quả quản lý hành chính công của tỉnh Long An, ngoài trừ nhóm "cơ sở vật chất". Nhân tố "đạo đức nghề nghiệp" và "nguồn nhân lực" có tương quan chặt với nhân tố phụ thuộc (Bảng 5). 


\section{Bảng 6}

Thống kê kiểm định phân tích nhân tố khẳng định (CFA)

\begin{tabular}{|l|c|c|}
\hline \multicolumn{1}{|c|}{ Chỉ số } & $\begin{array}{c}\text { Giá trị tính toán tù̀ } \\
\text { mô hình }\end{array}$ & $\begin{array}{c}\text { Giá trị yêu cầu theo Hair, } \\
\text { William, Babin, và } \\
\text { Anderson (2010) }\end{array}$ \\
\hline CMIN/DF & 2,585 & $<3$ \\
\hline CFI & 0,974 & $>0,900$ \\
\hline GFI & 0,927 & $>0,900$ \\
\hline AGFI & 0,951 & $>0,800$ \\
\hline RMSEA & 0,049 & $<0,100$ \\
\hline
\end{tabular}

Nguồn: Kết quả tính toán của tác giả

Tác giả thực hiện phân tích nhân tố khẳng định nhằm kiểm định sự phù hợp của các nhân tố tìm được (sau khi loại biến) với dữ liệu tổng thể. Phân tích CFA cho thấy, mô hình có hệ số chi bình phương trên bậc tự do $(\mathrm{CMIN} / \mathrm{DF})$ là $2,585 \leq 3$, như vậy mô hình phân tích có độ phù hợp cao với tổng thể (Hair et al., 2010). Bên cạnh đó, các chỉ số thống kê khác cũng cho thấy mô hình phù hợp với dữ liệu tổng thể (Bảng 6).

Kết quả kiểm định độ tin cậy của thang (đo FEA) và phân tích nhân tố khẳng định (CFA) cho thấy (Bảng 7), hệ số KMO thấp nhất là 0,605 (nhóm cơ sở vật chất), còn lại các nhóm khác đều có $\mathrm{KMO} \geq 0,8$, hệ số tải nhân tố, tổng phương sai trích đều đạt yêu cầu. Hệ số $\mathrm{CR}$ đều lớn hơn 0,7 . Điều này một lần nữa khẳng định các nhân tố trong mô hình và thang đo sử dụng trong nghiên cứu phù hợp với tổng thể.

\section{Bảng 7}

Phân tích độ tin cậy các thang đo (sau CFA)

\begin{tabular}{|c|c|c|c|c|c|c|c|}
\hline Nhân tố & Biến & $\begin{array}{c}\text { Hệ số tải } \\
\text { nhân tố }\end{array}$ & Eigenvalue & $\begin{array}{c}\text { Tổng } \\
\text { phương } \\
\text { sai trích } \\
(\%)\end{array}$ & KMO & $\begin{array}{c}\text { Barletts } \\
\text { Test }\end{array}$ & $\begin{array}{c}\text { Composite } \\
\text { reliability } \\
\text { (CR) }\end{array}$ \\
\hline CSVCF & $\begin{array}{l}\text { CSVC1 } \\
\text { CSVC2 } \\
\text { CSVC3 } \\
\text { CSVC4 }\end{array}$ & $\begin{array}{l}0,926 \\
0,925 \\
0,901 \\
0,902\end{array}$ & 1,804 & 83,516 & 0,605 & 0,000 & 0,771 \\
\hline QTTTF & $\begin{array}{l}\text { QTTT1 } \\
\text { QTTT2 } \\
\text { QTTT5 } \\
\text { QTTT6 } \\
\text { QTTT7 }\end{array}$ & $\begin{array}{l}0,757 \\
0,878 \\
0,840 \\
0,909 \\
0,907\end{array}$ & 3,698 & 73,955 & 0,872 & 0,000 & 0,919 \\
\hline NNLF & $\begin{array}{l}\text { NNL1 } \\
\text { NNL2 }\end{array}$ & $\begin{array}{l}0,915 \\
0,928\end{array}$ & 4,197 & 83,903 & 0,901 & 0,000 & 0,950 \\
\hline
\end{tabular}




\begin{tabular}{|c|c|c|l|c|l|l|c|}
\hline Nhân tố & Biến & $\begin{array}{c}\text { Hệ số tải } \\
\text { nhân tố }\end{array}$ & Eigenvalue & $\begin{array}{c}\text { Tông } \\
\text { phương } \\
\text { sai trích } \\
\text { (\%) }\end{array}$ & KMO & $\begin{array}{c}\text { Barletts } \\
\text { Test }\end{array}$ & $\begin{array}{c}\text { Composite } \\
\text { reliability } \\
\text { (CR) }\end{array}$ \\
\hline & NNL3 & 0,916 & & & & & \\
& NNL4 & 0,916 & & & & & \\
& NNL5 & 0,906 & & & & & \\
& DDNN1 & 0,849 & & & & & \\
& DDNN2 & 0,576 & & & & \\
& DDNN3 & 0,890 & & & & & \\
DDNNF & DDNN4 & 0,883 & 4,717 & 67,389 & 0,912 & 0,000 & 0,919 \\
& DDNN5 & 0,855 & & & & & \\
& DDNN6 & 0,873 & & & & & \\
& DDNN7 & 0,775 & & & & & \\
\hline \multirow{5}{*}{ HQQTF } & HQQT1 & 0,917 & & & & & \\
& HQQT2 & 0,926 & & & & & \\
& HQQT3 & 0,946 & 4,385 & 87,706 & 0,887 & 0,000 & 0,962 \\
& HQQT4 & 0,854 & & & & & \\
& HQQT5 & 0,939 & & & & & \\
\hline
\end{tabular}

Nguồn: Kết quả tính toán của tác giả

\subsection{Kết quả nghiên cứu bằng mô hình SEM}

\section{Bảng 8}

Tổng hợp kiểm định giả thuyết

\begin{tabular}{|l|c|c|c|c|}
\hline \multicolumn{1}{|c|}{ Nhân tố } & $\begin{array}{c}\text { Kỳ vọng } \\
\text { dấu }\end{array}$ & $\begin{array}{c}\text { Hệ số chuẩn } \\
\text { hóa }\end{array}$ & Giá trị P & $\begin{array}{c}\text { Kết quả kiểm } \\
\text { dịnh giả thuyết }\end{array}$ \\
\hline DDNNF ---> HQQTF & Dương & $0,336^{* * * *}$ & 0,000 & Chấp nhận \\
\hline QTTTF ---> HQQTF & Dương & $-0,225$ & 0,212 & Từ chối \\
\hline NNLF ---> HQQTF & Dương & $0,630^{* * *}$ & 0,000 & Chấp nhận \\
\hline CSVCF ---> HQQTF & Dương & $-0,006$ & 0,799 & Từ chối \\
\hline
\end{tabular}

Ghi chú: *** biểu thị $\mathrm{P}<1 \%$

Nguồn: Kết quả tính toán của tác giả

Thực hiện kiểm định mối quan hệ nhân quả giữa các nhân tố độc lập với nhân tố phụ thuộc, kết quả cho thấy: Hai nhân tố "đạo đức nghề nghiệp" và "nguồn nhân lực" có mối quan hệ nhân quả (tác động dương) với "hiệu quả quản trị hành chính công tại tỉnh Long An" với mức ý nghĩa $1 \%$. Hai nhóm nhân tố còn lại chưa tìm thấy mối quan hệ nhân quả với nhân tố phụ thuộc do chưa đạt mức ý nghĩa thống kê.

Sau kết quả kiểm định độ tin cậy của thang đo, tác giả thực hiện kiểm định mô hình lý thuyết bằng mô hình SEM nhằm kiểm định mối quan hệ nhân quả giữa các nhân tố trong mô 
hình. Kết quả cho thấy, mô hình nghiên cứu đề xuất là phù hợp thể hiện qua các chỉ số kiểm định: $\mathrm{CFI}=0,974, \mathrm{GFI}=0,927, \mathrm{AGFI}=0,951$ và $\mathrm{RMSEA}=0,049$ đều đạt chuẩn (Hair et al., 2010).

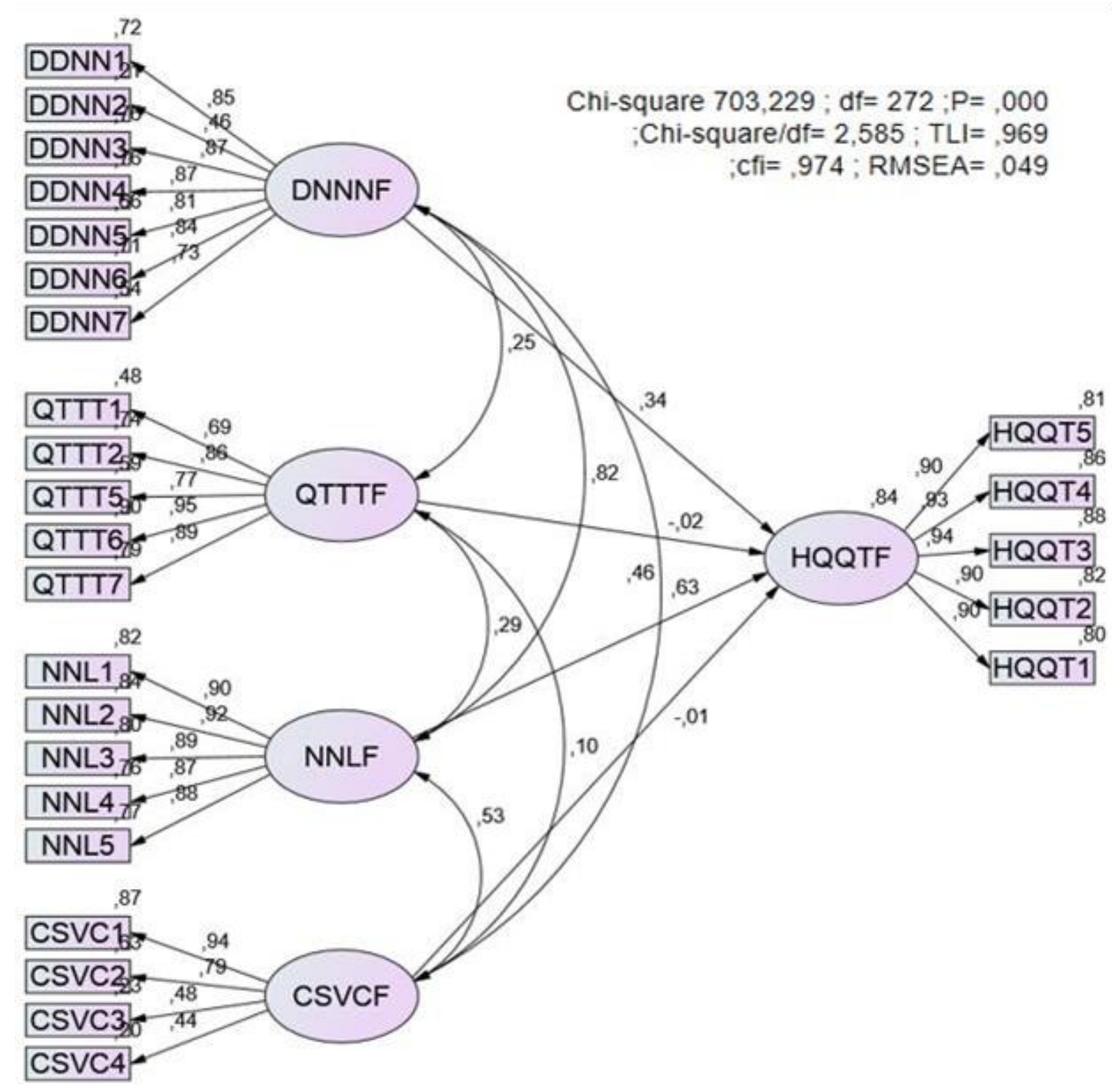

Hình 1. Mô hình SEM về hiệu quả quản trị hành chính công tại tỉnh Long An

Nguồn: Kết quả tính toán của tác giả

Kết quả nghiên cứu cho thấy, hiệu quả quản trị hành chính công tại tỉnh Long An chịu ảnh hưởng mạnh bởi nhóm "nguồn nhân lực" và "đạo đức nghề nghiệp". Những nhân tố khác được các đối tượng khảo sát cho rằng không phải là yếu tố quyết định đến hiệu quả quản trị hành chính công tại địa phương.

Hệ số hồi quy chuẩn hóa của nhóm nhân tố" nguồn nhân lực" đạt 0,63 cho thấy, ảnh hưởng mạnh của nhóm nhân tố này đến hiệu quả quản trị hành chính công tại tỉnh Long An. 
Hay nói cách khác, nguồn nhân lực (đội ngũ cán bộ công chức viên chức) có vai trò quyết định trong việc nâng cao hiệu quả quản trị hành chính công của địa phương (với mức ý nghĩa 1\%).

Hệ số hồi quy chuẩn hóa của nhóm “đạo đức nghề nghiệp đạt 0,34 (với mức ý nghĩa 1\%). Như vậy, đạo đức nghề nghiệp của cán bộ công chức viên chức có ảnh hưởng khá mạnh đến hiệu quả quản trị hành chính công tại tỉnh Long An.

Kết quả nghiên cứu phù hợp với một số nghiên cứu trước của: Ocampo (1998), Viện nghiên cứu đổi mới kinh tế Trung ương (2007), Pham (2012) và cũng phù hợp với thực tiễn. Trên thực tế, yếu tố con người và đạo đức nghề nghiệp vẫn luôn là yếu tố có ảnh hưởng mạnh đến hiệu quả làm việc của các cơ quan, chính quyền các cấp.

\section{Kết luận và khuyến nghị chính sách}

Bài nghiên cứu “đánh giá hiệu quả quản trị hành chính công tại tỉnh Long An” sử dụng phương pháp phân tích nhân tố khám phá (EFA), phân tích nhân tố khẳng định (CFA) và mô hình SEM từ mẫu 672 khảo sát thực nghiệm tại tỉnh Long An năm 2016. Kết quả nghiên cứu cho thấy, "nguồn nhân lực" và "đạo đức nghề nghiệp" của cán bộ công chức viên chức tại địa phương là hai nhân tố có ảnh hưởng mạnh, đóng vai trò quyết định đối với hiệu quả quản trị hành chính công của tỉnh Long An.

Từ kết quả phân tích dữ liệu nghiên cứu kết hợp với thực tiễn tại địa phương, tác giả đưa ra một số khuyến nghị về mặt chính sách để góp phần nâng cao hiệu quả quản trị hành chính công tại tỉnh Long An, thúc đẩy nhanh công tác cải cách hành chính của địa phương.

Nhóm nhân tố "nguồn nhân lực" có ảnh hưởng tích cực và ảnh hưởng mạnh đến hiệu quả quản trị hành chính công tại tỉnh Long An. Do đó, điều đầu tiên chính quyền tỉnh Long An cần thực hiện là nâng cao chất lượng nguồn nhân lực và hiệu quả làm việc của các cán bộ công chức viên chức của tỉnh. Cán bộ, công chức, viên chức cần được quan tâm đào tạo nâng cao trình độ chuyên môn nghiệp vụ, nắm vững kiến thức pháp luật; nâng cao tinh thần trách nhiệm và thực thi công vụ, có tinh thần tự giác cao, có kỹ năng và kỉnh nghiệm về chuyên môn tốt, có thái độ và hành vi ứng xử đúng mực, hành động vào làm việc theo quan điểm "lấy dân làm gốc, vì nhân dân phục vụ".

Bên cạnh đó, chính quyền tỉnh cần quan tâm đẩy mạnh hơn nữa việc bồi dưỡng, rèn luyện “đạo đức nghề nghiệp" của đội ngũ cán bộ công chức viên chức trong tỉnh; kiểm soát và xử lý nghiêm minh những hành vi sai trái, lạm dụng chức quyền, nhũng nhiễu, quan liêu, tiêu cực, ... để từ đó giúp nâng cao hơn nữa đạo đức công vụ của cán bộ công chức viên chức địa phương. Đây là tiền đề quan trọng giúp cho công tác cải cách hành chính, nâng cao hiệu quả quản lý hành chính của địa phương, cũng cố và nâng cao sự tin tưởng, sự hài lòng của người dân, doanh nghiệp đối với các cơ quan công quyền.

Chính quyền địa phương phải quan tâm cải tiến quy trình, thủ tục hành chính theo hướng tinh gọn, rút ngắn thời gian xử lý các thủ tục hành chính. Chính quyền cũng cần quan tâm thông tin công khai quy trình, thủ tục, tiến độ và kết quả xử lý hồ sơ hành chính trên các kênh thông tin khác như mạng internet, tin nhắn... nhằm tạo điều kiện thuận tiện để mọi người có nhu cầu có thể dễ dàng biết được. Trong giai đoạn hiện nay, bên cạnh việc quan tâm đầu tư, trang bị các phương tiện làm việc thuận tiện, đầu tư trang thiết bị và công nghệ giúp cán bộ, công chức, viên chức nâng cao hiệu quả giải quyết công việc, tạo thuận lợi cho người dân, doanh nghiệp trong hành chính, thì chính quyền tỉnh cần đẩy mạnh việc nâng cấp hệ thống thông tin điện tử nhằm công khai và áp dụng công nghệ để điện tử hóa quy trình xử lý thủ tục hành chính nhằm góp 
phần nâng cao hiệu quả quản trị hành chính, hướng đến chính quyền điện tử và phục vụ người dân được tốt hơn.

\section{Tài liệu tham khảo}

Bagozzi, R. P., \& Youjae, Y. (1989). On the use of structural equation models in experimental designs. Journal of Marketing Research, 26(3), 271-284.

Chính phủ. (2011). Nghị quyết ban hành "Chuơng trình tổng thể cải cách hành chính nhà nước giai đoạn 2011-2020”, Nghị quyết số 30c/NQ-CP, ban hành ngày 08/1 1/2011 [Resolution promulgating "Master Program of State Administration Reform in the 2011-2020 period", Resolution No. 30c / NQ-CP, issued on November 8, 2011]. Retrieved June 3, 2017, from https://thuvienphapluat.vn/van-ban/Bo-may-hanh-chinh/Nghi-quyet-30cNQ-CP-Chuong-trinh-tong-the-cai-cach-hanh-chinh-nha-nuoc-131576.aspx

Hair, J. F., William, C. B., Babin, B. J., \& Anderson, R. E. (2010). Multivarian data analysis, Englewood Cliffs, NJ: Prentice Hall.

Hoang Trong, \& Chu, N. M. N. (2008). Phân tích dĩ liệu nghiên cứu với SPSS [Analyze research data with SPSS]. Ho Chi Minh, Viet Nam: Nhà xuất bản Hồng Đức.

Hood, C. (1991). A public management for all seasons. Public Administration, 69(1), 3-19.

Hughes, O. E. (2012). Public management and administration (4th ed.), (pp.185-193). New York, NY: Palgrave Macmillan.

Hulland, J., Chow, Y. H., \& Lam, S. (1996). Use of causal models in marketing research: A review. International Journal of Research in Marketing, 13(2), 181-197.

Kaplan, R. S. (2005). How the balanced scorecard complements the McKinsey 7-S model. Strategy \& Leadership, 33, 41-46.

Le, H. T. V. (2006). Lý luận chung về hành chính công [General theory of public administration]. In H. N. Nguyen, Hành chính công (pp. 5-53). Hanoi, Vietnam: Nhà xuất bản Khoa học và Kỹ thuật.

MOPA. (2014). Areas of work in ministry of public administration. Retrieved June 4, 2017, from http://www.mju.gov.si/en/areas_of_work

Nedelko, Z. (2015). Ethics of managers in Slovenian public administration. Management, 20(2), 23-47

Nguyen, T. (2005). Kinh tế công cộng [Public Economy]. Hanoi, Vietnam: Nhà xuất bản Thống kê.

Nozharov, S. (2014). Model of effective management of bulgarian public administration managing EU funds. Economic Alternatives, 2014(4), 64-77.

Nozharov, S. (2017). Efficiency of public administration management in cultural heritage protection. Economic Alternatives, 2, 307-316 
Ocampo, R. B. (1998). Models of public administration reform: "New public management (NPM)". Asian Review of Public Administration, 10(1/2), 248-255.

Peter, F. D. (2016). Nhà quản trị thành công [Successful administrator] (H. D. Nguyen Trans.). Ho Chi Minh, Vietnam: NXB Pace \& NXB Trẻ.

Pham, T. D. (2012). Mối quan hệ giữa hành chính công và quản lí công [The relationship between public administration and public administration]. Hanoi, Vietnam: Liên hệ Việt Nam - Văn phòng Bộ Nội vụ.

Raadschelders, J. C. N. (2011). Public administration: The interdisciplinary study of government. Oxford, UK: Oxford University Press

Ralston, D. A., Egri, C. P., Reynaud, E., Srinivasan, N., Furrer, O., Brock, D., ... Wallace, A. (2011). A twenty-first century assessment of values across the global workforce. Journal of Business Ethics, 104(1), 1-31.

Robert, B. D., Janet, V. D., \& Tara, A. B. (2014). Public administration: An action orientation, Belmont (7th ed.). Belmont, CA: Thomson Wadsworth.

Schwartz, S. H. (1992). Universals in the content and structure of values - Theoretical advances and empirical tests in 20 countries. Advances in Experimental Social Psychology, 25, 165.

Steenkamp, J. B. E. M., \& VanTrijp, H. C. M. (1991). The use of LISREL in validating marketing constructs. International Journal of Research in Marketing, 8(4), 283-299.

Trung tâm Nghiên cứu Phát triển - Hỗ trợ Cộng đồng (CECODES). (2012). Giới thiệu về papi. Retrieved Retrieved June 13, 2017, from Kỹ thuật Việt Nam và Chương trình Phát triển Liên Hợp quốc (UNDP) tại Việt Nam website http://papi.org.vn/gioi-thieu-ve-papi

Ủy ban nhân dân tỉnh Long An. (2016). Báo cáo 40/BC-UBND ngày 15/3/2016, về việc thục hiện cải cách hành chính quý I, năm 2016 [Report 40 / BC-UBND dated March 15, 2016, on the implementation of the administrative reform quarter I, 2016]. Retrieved June 10, 2017, from https://www.quangbinh.gov.vn/3cms/bao-cao-tinh-hinh-thuc-hien-cong-tackiem-soat-thu-tuc-hanh-chinh-nam-2015-nhiem-vu-nam-2016-c.htm

Viện nghiên cứu đổi mới kinh tế Trung ương. (2007). Đổi mới cung úng dịch vu công ở Việt Nam [Innovating public service delivery in Vietnam]. Retrieved June 12, 2017, from https://tailieu.vn/doc/doi-moi-cung-ung-dich-vu-cong-o-viet-nam-69899.html 